УДК 004.94

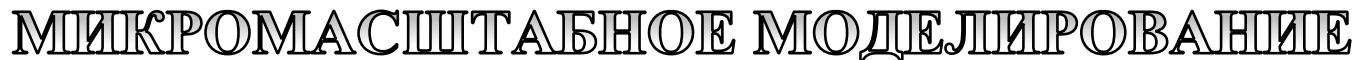

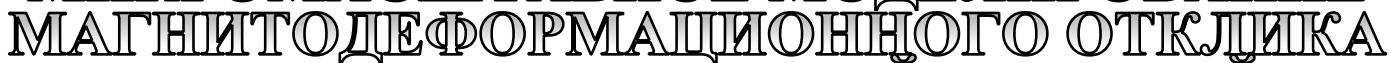

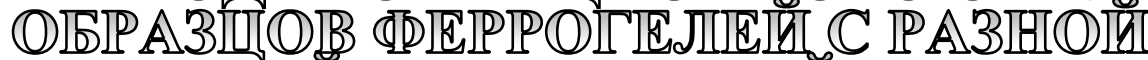

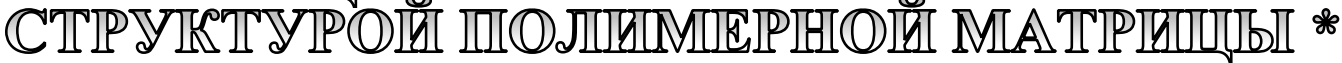

П.В. Меленев, Институт механики сплошных сред УрО РАН

А.В. Рыжков, Институт механики сплошных сред УрО РАН

\section{Для цитирования:}

Меленев П.В., Рыжков А.В. Микромасштабное моделирование магнитодеформационного отклика образцов феррогелей с разной структурой полимерной матрицы // Вестник Пермского федерального исследовательского центра. - 2021. - № 3. - C. 23-33. https://doi.org/10.7242/2658-705X/2021.3.4

Феррогели - это мягкие магнитно-активные композиционные материалы, матрицей в которых служат, как правило, гидрогели, а наполнителем фрерромагнитные коллоидные частицы. Ярко выраженная взаимосвязь магнитных и механических характеристик подобных материалов, а также их биосовместимость открывают возможность применения феррогелей в широком круге биомедицинских приложений. Сложность прямых наблюдений за процессами, происходящими на структурном масштабном уровне и играющими ключевую роль в формировании уникальных свойств феррогелей, повышает значение теоретических исследований внутреннего состояния материала.

В работе представлены результаты моделирования поведения малого образца феррогеля, полученные с применением метода крупнозернистой молекулярной механики. Матрица композита имитируется квазирегулярной сеткой идеальных полимерных цепей, а магнитные частицы включения, обладающие однодоменной структурой и одноосной магнитной анизотропией, помещаются в узлы этой сетки. Рассмотрено состояние образца в отсутствии внешнего воздействия, а также в циклах квазистатического намагничивания для двух вариантов топологии полимерной сетки (простой кубической и алмазоподобной), для различных величин магнитного момента частиц, их концентрации, а также энергии анизотропии.

Моделирование показало, что применение более мягкой матрицы с алмазоподобной структурой усиливает магитодеформационные эффректы. Наличие магнитной анизотропии приводит к возникновению дополнительных локальных напряжений при переориентации магнитных моментов, что затрудняет агрегирование частиц и намагничивание материала. Кроме того, обнаружено, что энергия анизотропии определяет характер изменения объёма образца в поле.

* Работа выполнена при поддержке проекта РФФИ № 17-41-590123 р_а проекта «Микромасштабное моделирование магнито-деформационного отклика образцов феррогелей». 
Ключевые слова: мягкий магнитный композит, феррогель, молекулярная динамика, управляемая доставка лекарств.

\section{Введение}

Феррогели (ФГ) представляют собой один из классов современных функциональных композитных материалов с ярко выраженной взаимосвязью магнитных и механических характеристик. Комбинация в составе ФГ высокоэластичной гелевой (гидрогелевой, во многих реальных образцах) матрицы и наполнения из ферромагнитных наноразмерных частиц позволяет получить биосовместимый материал, заметно реагирующий на магнитные поля даже сравнительно невысокой интенсивности, допустимые для применения in vivo [1] в таких задачах, как контролируемая доставка лекарств [2] или магнитная гипертермия раковых клеток [3]. А предельная мягкость гидрогелевой матрицы (чей упругий модуль составляет единицы кПа или менее) допускает значительное перемещение частиц включения, вызванное магнитными силами и способное привести к деформации или даже разрушению образца.

Эта особенность материала открывает дополнительный ряд потенциальных приложений ФГ: управляемое высвобождение лекарственных средств в заданной зоне организма [4], создание искусственных мускулов [5] и других тканей [6], восстановительная медицина [7] и др. Развитие этих и других областей применения ФГ требует понимания процессов, происходящих в материале под действием магнитных сил. Решение этой задачи осложняется тем, что прямое наблюдение за состоянием ФГ на структурном уровне до сих пор связано со значительными экспериментальными трудностями. В этих условиях теоретические и, в частности, численные исследования эволюции внутренней структуры ФГ могут быть чрезвычайно полезны для дальнейшего развития материалов этой группы.

К основным факторам, определяющим поведение ФГ, относятся способ включения частиц наполнителя в полимерную матрицу, топология полимерной сетки, под которой понимается пространственная структура полимерных сшивок, и тип этих сшивок. По последнему из свойств можно разделить полимерные гели на две основные группы. К первой из них относятся «физические» гели со сравнительно слабыми межцепочными сшивками, основанными на вандервальсовом взаимодействии сегментов, водородных связях либо слабом электростатическом взаимодействии. В гелях второго, «химического» типа для сшивок используются ковалентные связи, по энергии на несколько порядков превосходящие связи в «физических» гелях [8]. Это делает структуру сшивок у «химических» гелей значительно более стабильной. Магнитные наночастицы могут быть внедрены в гелевую матрицу как «физическим» [9], так и «химическим» [10] способами.

Во избежание сложностей, связанных с моделированием возможного разрушения слабых типов сшивок в процессе перемещения частиц и деформирования образца, мы сконцентрировали наше внимание на ФГ «химического» типа и считали структуру сшивок в материале неизменной. Большинство реальных образцов не обладают какой-то заданной регулярной топологией полимерной сетки. И есть теоретические работы, в которых сделана попытка воспроизвести случайную структуру сшивок. Например, в [11] эта структура возникает непосредственно в процессе расчёта. Получение качественных данных с применения подобных моделей требует рассмотрения образцов достаточно большого объёма либо усреднения по большому числу реализаций для сглаживания вклада неоднородностей в распределении сшивок. В нашей работе применен другой подход, заключающийся в сравнении результатов моделирования образцов с квазирегулярными полимерными сетками разных типов.

Как было отмечено выше, уникальные свойства ФГ во многом объясняются возможностью магнитоиндуцированных из- 
менений в распределении наполнителя материала. Поэтому одной из задач данной работы было наблюдение за формированием кластеров частиц включения под действием магнитных сил. Кроме того, исследовалось изменение объёма образца при намагничивании - свойства, важного, например, для задачи контролируемого высвобождения лекарственных средств. Рассматривалось поведение образцов ФГ с разными значениями концентрации наполнителя, магнитного момента частиц и пороговой энергии магнитной анизотропии.

\section{Модель}

В основе нашей модели лежит так называемый подход крупнозернистой молекулярной динамики (coarse-grained molecular dynamic), согласно которому при моделировании выбирается уровень детальности описания рассматриваемой системы, достаточный для воспроизведения наиболее важных, с точки зрения исследования, процессов. Прочие же аспекты поведения системы описываются упрощенно либо не учитываются в модели. Данный подход уже был успешно применен для численного исследования магнитополимерных композитов [12].

Предполагается, что в рассматриваемых условиях ни взаимодействия внутри материала, ни внешние воздействия не могут менять внутренней структуры полимерных макромолекул и магнитных наночастиц. Поэтому были выбраны следующие модели элементов композита без детализации их строения. Полимерные фрагменты макромолекул между узлами сетки описываются в виде цепей из одинакового числа жёстких сегментов - «блобов» диметром $d_{\mathrm{b}}$, связи между которыми описываются гармоническим потенциалом

$$
U^{\mathrm{el}}\left(r_{i}, r_{j}\right)=K^{\mathrm{el}}\left(\left|r_{i}-r_{j}\right|-l_{0}\right)^{2},
$$

где $r_{i}, r_{j}$ - это радиус-векторы центров $i$-го и $j$-го блобов, $K^{\mathrm{el}}-$ упругий модуль, $l_{0}=d_{\mathrm{b}}-$ равновесная длина связи.

Упругие связи с потенциалом (1) применяются и для соединения полимерных цепей с магнитными частицами, но в этом случае $r_{j}$ представляет собой радиус-вектор точки крепления на поверхности частицы.

Магнитное включение тоже представлено в виде жёстких сфер одинакового диаметра $d_{\mathrm{p}}=3 d_{\mathrm{b}}$. Частицы имеют однодоменную магнитную структуру, то есть их магнитные моменты сохраняют постоянную величину $\mu$, но могут менять своё направление. Кроме того, частицы обладают одноосной магнитной анизотропией с заданной пороговой энергией $E_{\mathrm{A}}$. Предполагается, что частицы взаимодействуют между собой, как точечные диполи. Таким образом, магнитная часть энергии $k$-й частицы включения имеет вид

$$
\begin{aligned}
& U_{k}^{\text {magn }}=-\mu_{0} \mu \mathbf{e}_{k} \cdot \mathbf{H}_{\mathbf{0}}-E_{\mathrm{A}}\left(\mathbf{e}_{k} \cdot \mathbf{n}_{k}\right)^{2}+ \\
& +\frac{\mu_{0}}{4 \pi} \mu^{2} \sum_{i, i \neq k}^{N^{\text {magn }}}\left(\frac{\mathbf{e}_{i} \cdot \mathbf{e}_{k}}{r_{i k}{ }^{3}}-\frac{3\left(\mathbf{e}_{i} \cdot \mathbf{r}_{i k}\right)\left(\mathbf{e}_{k} \cdot \mathbf{r}_{i k}\right)}{r_{i k}{ }^{3}}\right),
\end{aligned}
$$

где $\mu_{0}-$ магнитная восприимчивость вакуума, $\mathbf{H}_{0}-$ внешнее магнитное поле, $\mathbf{e}_{k}$, $\mathbf{n}_{k}$ - орты направлений магнитного момента и оси лёгкого намагничивания частицы, соответственно; $\boldsymbol{N}^{\text {magn }}$ - общее число магнитных частиц в системе.

Полимерные цепи крепятся к магнитным частицам в точках их поверхности, выбранных в соответствии с топологией полимерной сетки. Таким образом, матрица деформируется как при перемещении, так и при вращении частиц включения. При этом, сама матрица может влиять и на поступательные, и на вращательные степени свободы частиц.

Стерические взаимодействия элементов модели - частиц и «блобов» - имитируются на основе ограниченного потенциала Леннарда-Джонса (ЛЖ):

$$
\begin{gathered}
U^{\sqcup}\left(r_{i j}\right)=\left\{\begin{array}{c}
4 \varepsilon\left[\left(\frac{s}{r_{i j}}\right)^{12}-\left(\frac{s}{r_{i j}}\right)^{6}+\frac{1}{4}\right], \\
0,
\end{array}\right. \\
0 \leq r_{i j} \leq R_{\text {cutoff }} \\
r_{i j}>R_{\text {cutoff }}
\end{gathered}
$$

здесь $r_{i j}=\left|\mathbf{r}_{j}-\mathbf{r}_{i}\right|-$ расстояние между центрами элементов модели, 
$R_{\text {cutoff }}=\frac{1}{2}\left(d_{i}+d_{j}\right)-$ полусумма их диаметров, $\varepsilon$ и $s=2^{-1 / 6} R_{\text {cutoff }}$ - соответственно, энергетический и пространственный параметры потенциала Леннард-Джонса.

Процесс моделирования заключается в численном интегрировании уравнений движения всех элементов модели. Наличие тепловых колебаний магнитных и пространственных степеней свободы, существенное для рассматриваемых процессов, происходящих на субмикронном масштабе при комнатной температуре, учитывается включением в уравнения движения слагаемых, описывающих действие термостата Ланжевена с заданной постоянной температурой $T$. Отношение характерных магнитных энергий системы к энергии теплового движения задаёт значения следующих безразмерных параметров модели:

$$
\lambda=\frac{\mu_{0}}{4 \pi} \frac{\mu^{2}}{d_{\mathrm{p}}{ }^{3} k_{\mathrm{B}} T}, \quad \xi=\mu_{0} \frac{\mu H_{0}}{k_{\mathrm{B}} T}, \quad \sigma=\frac{E_{\mathrm{A}}}{k_{\mathrm{B}} T},
$$

где величины $\lambda, \xi, \sigma$ отражают, соответственно, интенсивность дипольного взаимодействия двух частиц, энергию Зеемана взаимодействия магнитного момента частицы с внешним полем и энергию магнитной анизотропии частицы.

Детальное описание модели можно найти в нашей работе [13], где также вычислены значения параметров модели в размерных величинах и показано, что они согласуются с экспериментальными данными по ФГ с гидрогелевой матрицей. В целом эта работа посвящена описанию поведения образцов ФГ с квазикубической структурой полимерной сетки. Результаты расчётов показали, что выбранный тип топологии матрицы приводит к достаточно высокой жёсткости образцов. Для того чтобы усилить магнитодеформационные эффекты, было принято решение рассмотреть материал с меньшей плотностью сшивок, а именно, обладающий полимерной сеткой с алмазоподобной структурой. В этом случае каждый узел сетки связывает 4 полимерные цепочки, а не 6 , как в варианте простой ку- бической структуры. В одни узлы сетки помещаются магнитные частицы, в другие - полимерные «блобы». Доля «магнитных» узлов, обозначенная как с, отражает концентрацию магнитного наполнителя в композите. Образец может включать до 1000 магнитных частиц, в этом случае все узлы полимерной сетки являются «магнитными» и $\mathrm{c}=1,0$. В данной работе представлены результаты для $\mathrm{c}=0,2 \ldots 0,8$, что соответствует объёмной доле частиц $\varphi$ от 0,6 до 5\%об. Этот диапазон значений $\varphi$ согласуется с данными реальных ФГ [14, 15$]$.

Для количественной оценки наличия магнитных кластеров в материале в нашей работе используется параметр агрегирования $q_{n}$, равный доле магнитных частиц образца, имеющих по крайней мере $n$ магнитных частиц в ближайшем окружении (в наших расчётах радиус поиска задавался равным $\left.1,1 q_{\mathrm{p}}\right)$. Это означает, что значение $q_{1}$ отражает долю частиц, участвующих в любых агрегатах; $q_{2}$ уже не учитывает частицы, включённые в изолированные пары и расположенные на концах цепей; $q_{3}$ включает в себя лишь участки ветвления и объёмные кластеры, но не частицы внутри изолированных цепей.

\section{Результаты}

Первым этапом работы было исследование зависимости состояния образцов ФГ в отсутствии внешних нагрузок от характеристик частиц и топологии матрицы. На втором этапе расчётов рассматривалось поведение материала в циклах квазистатического намагничивания, имитируемых следующим образом: однородное магнитное поле было направлено вдоль оси $0 \mathrm{z}$ декартовой системы координат, а его напряжённость ступенчато увеличивалась от 0 до максимального значения $H_{\max }$, а затем уменьшалась обратно до нуля. Между моментами изменения поля проводилось число расчётных шагов, достаточное для получения состояния, близкого к равновесному. Расчёты проводились с использова- 
нием пакета ESPResSo [16] на компьютерном кластере Triton вычислительного центра ПФИЦ УрО РАН.

Результаты расчетов без поля. Для оценки роли дипольного магнитного взаимодействия в формировании внутренней структуры ФГ в исходном, ненамагниченном состоянии были получены зависимости значения $q_{n}$ от $\lambda$ при различных величинах энергии анизотропии. Отметим, что во всех проведённых расчётах, как без поля, так и в его присутствии, $q_{n}$ $\approx 0$ для $\mathrm{n} \geq 3$. Это означает, что в рассматриваемых вариантах образцов ФГ отсутствуют кластеры топологически более сложные, чем пары и отдельные цепи.

На рис. 1 представлены результаты расчёта $q_{1}$ и $q_{2}$ для предельных значений и концентрации частиц $(\mathrm{c}=0,2,0,8)$, и энергии анизотропии $(\sigma=0, \infty)$. В отсутствии внешних нагрузок дипольное взаимодействие является единственной движущей силой перемещения магнитных частиц, и в образцах с высокой концентрацией частиц и/или более высоким значением параметра $\lambda$ (отражающим величину магнитного момента частиц) бо́льшая доля частиц включается в формирование агрегатов. Магнитная анизотропия, напротив, затрудняет кластеризацию, так как наличие лёгкой оси намагничивания у частицы приводит к тому, что переориентация магнитного момента может повлечь за собой поворот «тела» частицы. А это, в свою очередь, может при-

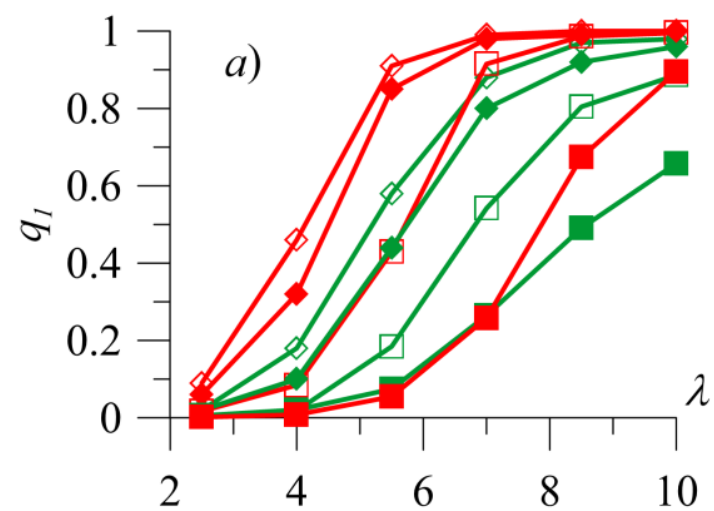

вести к возникновению механических напряжений в прилегающей матрице даже в отсутствии трансляционных перемещений частицы. Как следствие, в образцах, наполненных частицами с высоким значением энергии анизотропии, магнитоиндуцируемые деформации требуют большей интенсивности магнитных взаимодействий, то есть большей величины магнитного момента частицы и внешнего поля (значениями параметров $\lambda$ и $\xi$, соответственно).

Расчёты вариантов модели с разной топологией полимерного связующего ожидаемо показали, что в образцах с алмазоподобной структурой сетки (на графиках данные для этого типа матрицы отмечены ромбами) агрегирование частиц проходит более активно, чем в материале с «кубической» сеткой (квадратики на графиках). Для примера посмотрим на зависимость $q_{2}$, представленную на рис. 1, б: в образцах с алмазоподобной структурой связующего, высокой концентрацией наполнителя и значением $\lambda \geq 5,5$ от $50 \%$ и более частиц входят в цепи (рис. 2, $a$ ). А при $\lambda \geq 8,5$ этот уровень кластеризации достигается даже в образцах с низкой концентрацией частиц. В то же время в материале $\mathrm{c}$ кубической топологией полимерной сетки схожие значения $q_{2}$ демонстрируют только высоконаполненные образцы и лишь при магнитоизотропных частицах (рис. 2).

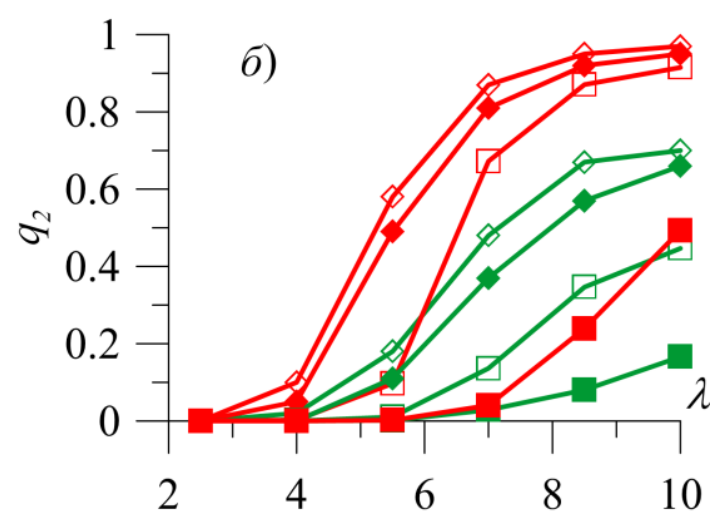

Рис. 1. Зависимость параметров агрегирования $q_{1}, q_{2}$ от параметра дипольных взаимодействий

ג. Цвет графиков соответствует концентрации частий: зелёный $-c=0,2$, красный $-c=0,8$.

Тип символов указывает на структуру матрииьл: ромбы - алмазоподобную, квадраты -с квазикубическую. Пустыли символами отмечены результаты магнитоизотропных частич (то есть $\sigma=0)$, а закрашенными - случай $\sigma=0$ 


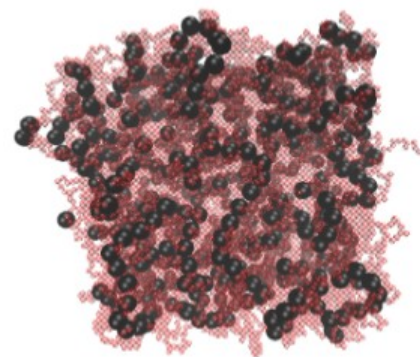

a) $\xi=0$ $\lambda=5.5$

$\mathrm{c}=0.8$

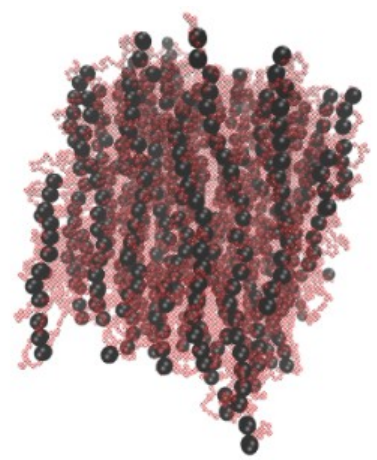

б) $\xi=15$

Рис. 2. Примеры состояний образиов феррогеля с высокой интенсивностью магнитных взаимодействий частии в отсутствии (а) либо в присутствии (б) внешнего магнитного поля

\section{Результаты расчетов в поле}

Моделирование циклов намагничивания ФГ не выявило заметных проявлений гистерезисного поведения у рассмотренных образцов. Поэтому на приводимых ниже графиках полевых зависимостей не показаны ветки, соответствующие размагничиванию образцов.

Степень намагниченности материала внешним полем, интенсивность которого измерялась в единицах $\xi$, характеризовалась усредненной по образцу величиной компоненты магнитного момента частицы, параллельной полю и отнесённой к модулю магнитного момента $-\left\langle\mu_{z}\right\rangle / \mu$. Соответствующая зависимость представлена на рис. 3. Видно, что межчастичные магнитные взаимодействия (чья средняя интенсивность растёт вместе с концен- трацией частиц и/или величиной $\lambda$ ) могут влиять на процесс намагничивания противоположным образом в зависимости от энергии анизотропии частиц. Так, при $\sigma=\infty$ межчастичные магнитные силы затрудняют намагничивание и соответствующие графики лежат ниже кривой функции Ланжевена, описывающей намагничивание ансамбля невзаимодействующими магнитоизотропных частиц. А при нулевом значении $\sigma$, напротив, в образцах с более интенсивными магнитными взаимодействиями моменты частиц легче ориентируются внешним полем, а зависимости $\left\langle\mu_{z}\right\rangle / \mu$ от $\xi$ лежат выше кривой функции Ланжевена.

Данный эффект можно объяснить тем, что как было отмечено выше, в образцах, наполненных частицами с высокой энер-

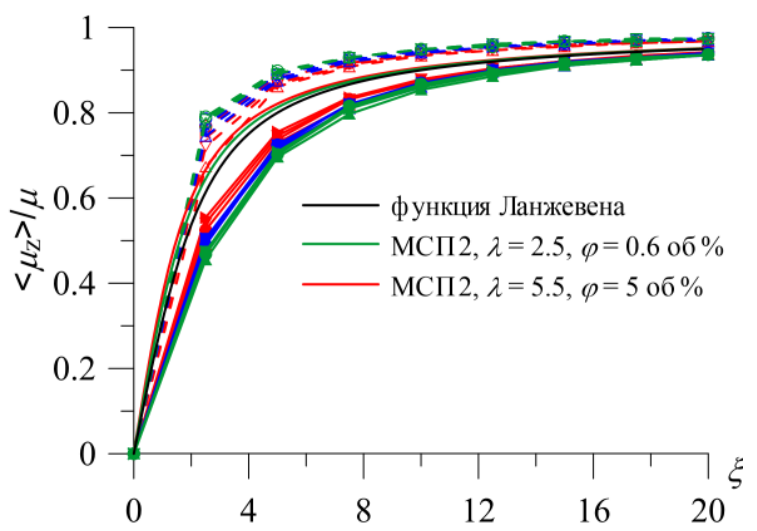

Рис. 3. Зависимость проекции среднего магнитного момента на направлении внешнего поля от его величины (выраженной в единицах $)$ ) для образиов феррогеля с разными параметрами наполнителя. Цвет графиков указывает на интенсивность дипольного взаимодействия частиц: зелёныц $-\lambda=2,5$, синий $-\lambda=4,0$, красный $-\lambda=5,5$. Тип символов соответствует концентрации частии: $\bullet-c=0,2, \boldsymbol{-}-c=0,35, \boldsymbol{\nabla}-c=0,5, \boldsymbol{\Delta}-c=0,8$. Пустые символь и иттриховые линии соответствуют результатам магнитоизотропных частии (то есть $\sigma=0)$, а закрашенные символы и сплошные линии - случаю $\sigma=\infty$ 
гией анизотропии, переориентация магнитного момента вызывает поворот самой частицы и, соответственно, упругий ответ окружающей её матрицы. Причём эти локальные напряжения дополнительно затрудняют и трансляционные перемещения частиц, в том числе и способствующие намагничиванию материала: формирование новых либо достраивание уже существующих магнитных кластеров, их ориентирование вдоль поля. А в случае низких значений $\sigma$, в отсутствии описанных выше магнитоиндуцированных локальных деформаций, материал обладает эффективно более мягкой матрицей, которая допускает более свободную перестройку структуры наполнителя, в частности, ориентацию цепей частиц по внешнему полю (см. рис. 2, б).

Это объяснение подтверждается данными о зависимости от $\xi$ параметров агрегирования, представленными на рис. 4 . $\mathrm{У}$ образцов с $\sigma=\infty$ значения $q_{1}, q_{2}$ растут вместе с полем. В то же время в образцах с магнитоизотропным наполнителем наблюдается некоторое снижение параметра агрегирования при намагничивании. Это можно объяснить тем, что в отсутствии поля агрегаты расположены произвольным образом (см. рис. $2, a$ ), и существенная часть частиц входит в фрагменты цепей, ориентированные неколлинеарно к направлению внешнего поля (оси $\mathrm{Oz}$ ). И возможны случаи, когда при на-

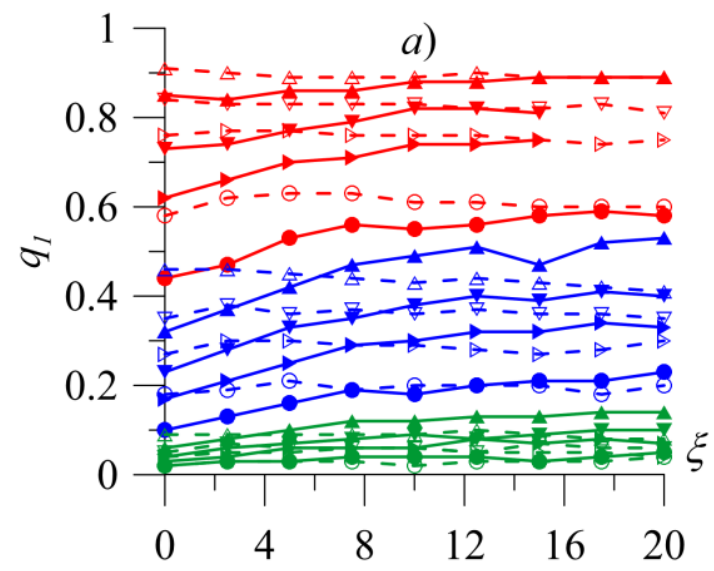

магничивании энергетически более выгодно не выстраивать искривлённый агрегат по полю целиком, а разорвать его и ориентировать части по отдельности. При этом некоторые частицы, оказавшиеся в «невыгодных» фрагментах, при разрыве агрегата могут не войти ни в один из образовавшихся кластеров, что приводит к уменьшению значения $q_{1}$.

Дополнительную информацию о роли упругого связующего ФГ в формировании магнитного отклика материала можно получить из сравнения наших результатов с данными о статическом намагничивании аналогичных по наполнителю магнитных жидкостей с вязкой несущей средой. Такие равновесные кривые намагничивания были построены на основе так называемого модифицированного метода среднего поля второго порядка (МСП2). Этот теоретический подход, предложенный А.О. Ивановым и О.Б. Кузнецовой и представлен, например, в работе [17], хорошо описывает экспериментальные данные для широкого диапазона концентраций магнитных жидкостей.

На рис. 3 представлены две кривые, построенные согласно МСП2 для систем, соответствующих по величине магнитного момента и средней концентрации частиц вариантам наших образцов с наименьшей $(c=0,2$, то есть $\varphi=0,6 \%$ об, и $\lambda=2,5)$ и наибольшей $(c=0,8$, то есть $\varphi=5 \%$ об, и $\lambda=5,5)$ интенсивностью дипольных взаимодейст-

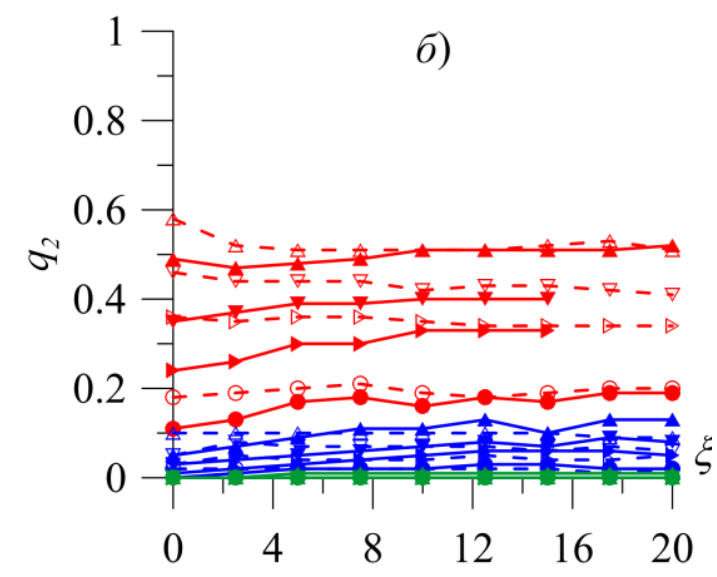

Рис. 4. Зависимость параметров агрегирования $q_{1}, q_{2}$ от величины внешнего поля, выраженной в единицах $\xi$, для образиов феррогеля с разными параметрами наполнителя.

Цвета, типы символов и линий имеют то же значение, что и на рис. 3 
вий. Видно, что оба графика лежат над кривой Ланжевена - с той же «стороны», что и расчётные данные образцов с $\sigma=0$. Отметим, что магнитная анизотропия частиц не влияет на равновесные значения намагниченности магнитной жидкости, что видимо и делает сходным магнитное поведение частиц в них и в феррогелях с магнитоизотропным наполнителем. В то же время кривые МСП2 находятся ближе к кривой Ланжевена, чем любая из полученных нами для образов ФГ. Последнее наблюдение можно объяснить тем, что упругая матрица композита частично демпфирует тепловые колебания частиц и тем самым стабилизирует магнитные агрегаты, наличие которых усиливает магнитный отклик системы.

Одной из областей применения ФГ является разработка систем адресной доставки и высвобождения лекарственных средств. Например, можно предложить следующую схему: образец субмикронных размеров наполняется водным раствором препарата; полученный контейнер запускается в кровеносную систему, возможно с применением внешнего магнитного поля для управления движения ФГ в ней. А в заданной области организма вызывается магнитоиндуцированное сжатие образца и высвобождение препарата [18].

Было исследовано изменение объёма образцов ФГ во внешнем поле, соответствующие результаты представлены на рис. 5. Расчёты подтвердили наличие магнитодеформационного эффекта, причем ожидаемо у материала с алмазоподобной структурой полимерной сетки он проявляется ярче. Более того, образцы с этим типом связующего демонстрируют как сжатие, так и разбухание в поле, в зависимости от энергии анизотропии частиц наполнителя. Так, объём образца с $\sigma=\infty$ при намагничивании уменьшается. В случае материала с тем же наполнителем, но кубической структурой сетки, также наблюдается сжатие образцов, но менее заметное. Отметим, что аналогичное поведение было обнаружено экспериментально на образцах ФГ, наполненных наночастицами магнетита $[19,20]$.

При моделировании материала с магнитоизотропными частицами у образцов с кубической структурой полимерного связующего наблюдается либо практически нуле-

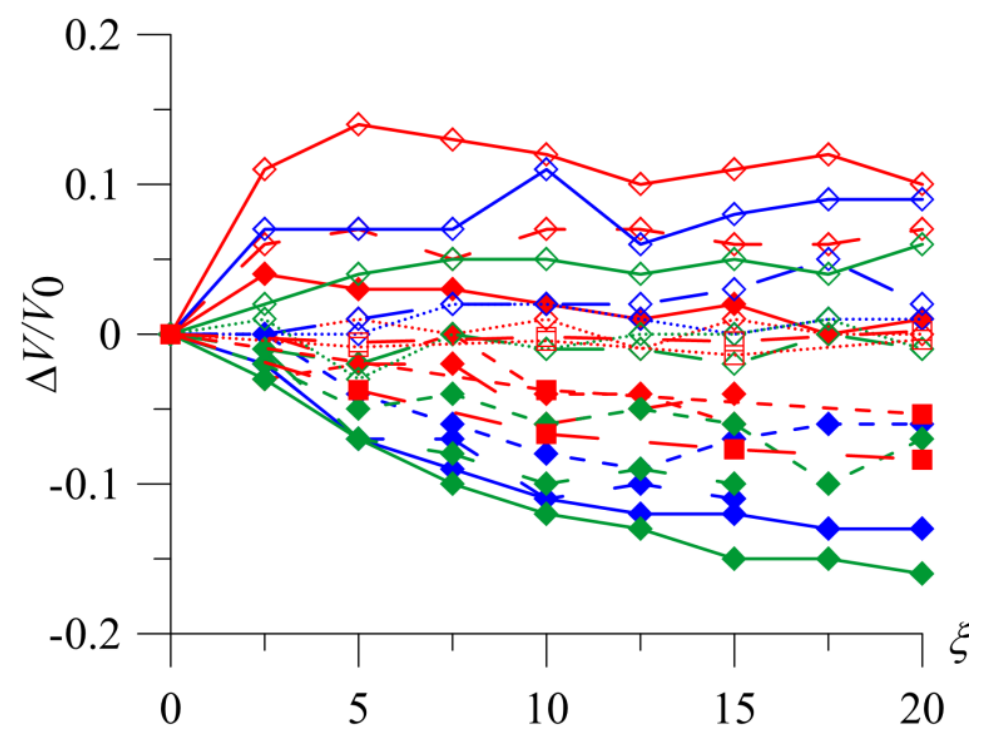

Рис. 5. Зависимость изменения объёма образца при намагничивании от величины внешнего поля, выраженной в единицах $\xi$, для образцов феррогеля с разными параметрами наполнителя. Цвет графиков указывает на интенсивность дипольного взаимодействия частии:: зелёный $-\lambda=2,5$, синий $-\lambda=4,0$, красный $-\lambda=5,5$. Тип символов указывает на структуру матрицы: ромбы - алазоподобную, квадраты - с квази-кубическую. Пустые символь и штриховые линии соответствуют результатам магнитоизотропных частии (то есть $\sigma=0)$, а закрашенные символь и сплошные линии - случаю $\sigma=\infty$. Тип линий указывает на концентрацию частиц наполнителя: шттиховые соответствуют $c=0,35$, длинный штрих $-c=0,5$, сплошные $-c=0,8$ 
вое изменение объёма, либо его слабое (по сравнению со случаем $\sigma=\infty$ ) уменьшение. При этом образцы с алмазоподобной топологией сетки демонстрируют в поле заметное разбухание при всех рассмотренных значениях концентрации частиц и величины магнитного момента. У образцов с более интенсивными магнитными взаимодействиями объём меняется сильнее. Таким образом, можно предположить, что в случае достаточно мягкой гидрогелевой матрицы применение наполнителей, отличающихся по энергии анизотропии, позволяет получить образцы ФГ с разнообразным магнитодеформационным поведением.

\section{Заключение}

В работе проведено численное исследование магнитомеханического отклика малого образца феррогеля на внешнее постоянное магнитное поле. В модели, базирующейся на подходе групнозернистой молекулярной динамики, полимерное связующее имитируется системой идеальных полимерных цепей (состоящих из жёстких сфер с гармоническими связями между ними), объединённых в квазирегулярную структуру. Монодисперсный магнитный наполнитель состоит из сферических частиц, обладающих однодоменной структурой и одноосной анизотропией магнитных свойств. Магнитные частицы размещаются в узлах полимерной сетки, а для последней рассмотрено два варианта топологии, отличающиеся средней плот- ностью полимерных сшивок. Расчёты показали, что матрица с алмазоподобной структурой допускает более значительные магнитоиндуцированные перемещения частиц включения (в частности, кластерообразование) по сравнению с вариантом простой кубической сетки. В результате, образцы с алмазоподобной структурой связующего демонстрируют более яркое проявления магнитодеформационного эффекта, в частности, изменение объёма образцов в поле.

В образцах с более высокой энергией магнитной анизотропии частиц перемагничивание моментов частиц затрудняется упругой реакцией матрицы на поворот частиц. Кроме того, наличие подобных локальных напряжений приводит к эффективному упрочнению матрицы, которое затрудняет трансляционные перемещения частиц, а тем самым, осложняет и намагничивание образца, и кластерообразование в материале, и деформации образца в поле. При этом образцы, наполненные вызокоанизотропными частицами, демонстрируют магнитоиндуцированное сжатие (уменьшение объёма), в то время как в случае магнитоизотропного наполнителя наблюдается противоположный эффект образцы разбухают в поле. Данный эффект может быть полезен при проектировании и развитии реальных приложений феррогелей, таких как создание контейнеров для контролируемой доставки и высвобождения малых доз препаратов.

\section{Библиографический список}

1. Guisasola E., Vallet-Regi M., Baeza A. Magnetically responsive polymers for drug delivery applications // Stimuli Responsive Polymeric Nanocarriers for Drug Delivery Applications. - Vol. 1. Cambridge: Woodhead Publishing, 2018. - P. 143-168.

2. Zhao X., Kim J.,Cezar C.A. [et. al.] Active scaffolds for on-demand drug and cell delivery // Proceedings of National Academy of Science of the USA. - 2011. - Vol. 108. - P. 67-72.

3. Babincova M., Leszczynska D., Sourivong P. [et. al.] Superparamagnetic gel as a novel material for electromagnetically induced hyperthermia // Journal of Magnetism and. Magnetic Materials. - 2001.Vol. 225. - P. 109-112.

4. Lin C.C., Metters A.T. Hydrogels in controlled release formulations: network design and mathematical modeling // Advanced Drug Delivery Reviews. - 2006. - Vol. 58. - P. 1379-408.

5. Shahinpoor M., Kim K.J., Mojarrad M. Artificial Muscles: Applications of Advanced Polymeric Nanocopmosites. - New York, London: CRC Press, 2007. - 480 p.

6. Li Y., Huang G., Zhang X. [et. al.] Magnetic Hydrogels and Their Potential Biomedical Applications // Advanced Functional Materials. - 2013. - Vol. 3. - P. 660-672. 
7. Blyakhman, F.A., Safronov A.P., Zubarev A.Yu. [et. al.] Polyacrylamide ferrogels with embedded maghemite nanoparticles for biomedical engineering // Results in Physics. - 2017. - Vol. 7. - P. 3624-3633.

8. Philippova O.E. Responsive Polymer Gels // Polymer Science. Series C. - 2000 - Vol. 42. - P. 23282352.

9. Ramanujan R., Lao L. The mechanical behavior of smart magnet-hydrogel composites // Smart Materials and Structures. - 2006. - Vol. 15. - P. 952.

10. Ilg P. Stimuli-responsive hydrogels cross-linked by magnetic nanoparticles // Soft Matter. - 2013. Vol. 9. - P. 3465

11. Minina E.S., Sánchez P.A., Likos C.N., Kantorovich S.S. The influence of the magnetic filler concentration on the properties of a microgel particle: Zero-field case // Journal of Magnetism and. Magnetic Materials. - 2018. - Vol. 459. - P. 226-230.

12. Weeber R., Hermes M., Schmidt A.M., Holm C. Polymer architecture of magnetic gels: a review // Journal of Physics: Condensed Matter. - 2018. - Vol. 30. - P. 063002.

13. Ryzhkov A.V., Melenev P.V., Balasoiu M., Raikher Yu.L. Structure organization and magnetic properties of microscale ferrogels: The effect of particle magnetic anisotropy // Journal of Chemical. Physics. - 2016. - Vol. 145. - P. 074905.

14. Galicia J.A., Cousin F., Dubois E. [et. al.] Static and dynamic structural probing of swollen polyacrylamide ferrogels // Soft Matter.- 2009. - Vol. 13. - P. 2614-2624.

15. Campbell S.B., Patenaude M., Hoare T. Injectable superparamagnets: highly elastic and degradable poly(N-isopropylacrylamide)-superparamagnetic iron oxide nanoparticle (SPION) composite hydrogels // Biomacromolecules. - 2014. - Vol. 14. - P. 644-653.

16. Arnold A., Lenz O., Kesselheim S. [et. al.] ESPResSo 3.1 - Molecular Dynamics Software for CoarseGrained Models // Meshfree Methods for Partial Differential Equations. - 2013. - Vol. 6. - P. 1-23.

17. Ivanov A.O., Kuznetsova O.B. Magnetic properties of dense ferrofluids: An influence of interparticle correlations // Physical Review E. - 2001. - Vol. 64. - P. 041405.

18. Liи T.-Y., Ни S.-H., Liи D.-M. [et. al.] Biomedical nanoparticle carriers with combined thermal and magnetic responses // Nano Today. - 2009. - Vol. 4. - P. 52-65.

19. Liu T.-Y., Hu S.-H., Liu T.-Y. [et. al.] Magnetic-Sensitive Behavior of Intelligent Ferrogels for Controlled Release of Drug // Langmuir. - 2006. - Vol. 22. - P. 5974-5978.

20. Qin J., Asempah I., Laurent S. [et. al.] Injectable Superparamagnetic Ferrogels for Controlled Release of Hydrophobic Drugs // Advanced Materials. - 2009. - Vol. 21. - P. 1354-1357.

\title{
MICROSCALE MODEL OF FERROGEL SAMPLES WITH DIFFERENT POLYMER MATRIX STRUCTURE
}

\author{
P.V. Melenev, A.V. Ryzhkov \\ Institute of Continuous Media Mechanics UB RAS
}

\section{For citation:}

Melenev P.V., Ryzhkov A.V. Microscale model of ferrogel samples with different polymer matrix structure // Perm Federal Research Center Journal. - 2021 - № 3. - P. 23-33. https://doi.org/10.7242/2658-705X/2021.3.4

Ferrogels is a group of soft magnetically active composite materials with polymer gel (generally hydrogel) matrix filled by ferromagnetic colloid particles. Pronounced interrelation between magnetic and mechanical properties of ferrogels together with their biocompatibility make ferrogels attractive for various bio-medical applications. Evolution of ferrogel internal structure plays pivotal role in formation of unique magnetic mechanical behavior of ferrogel. Difficulty of direct observation on the structure scales enhances importance of theoretical (and numerical) study on ferrogels.

The paper presents the results of modeling the behavior of a small ferrogel sample (up to 1000 magnetic particles) obtained using the method of coarse-grained molecular dynamics. The composite matrix is imitated by a quasi-regular mesh of ideal spring-beads chains, and single-domain magnetic particles with uniaxial anisotropy are placed in nodes of this mesh. The state of the sample is considered in the absence of external action, as well as in cycles of quasi-static magnetization for two 
variants of the topology of the polymer network (simple cubic and diamond-like), for different values of the magnetic moment of particles, their concentration, and anisotropy energy.

Simulation has shown that samples with softer matrix (i.e. of diamond-like structure) demonstrate more noticeable magnetic deformation effects. The presence of magnetic anisotropy excites additional local stresses during particle moment reorientations, which complicates particles clustering and sample magnetization. Moreover, it has been found that anisotropy energy determines the character of the field induced change in the sample volume.

Keywords: soft magnetic composite, ferrogel, molecular dynamics, targeted drug delivery.

\section{Сведения об авторах}

Меленев Петр Викторович, кандидат физико-математических наук, Институт механики сплошных сред УрО РАН - филиал Пермского федерального исследовательского центра УрО РАН («ИМСС УрО РАН»), 614013, ул. Академика Королева, 1; e-mail: melenev@icmm.ru

Рыжкков Александр Владимирович, кандидат физико-математических наук, «ИМСС УрО РАН»; e-mail: ryzhkovalexandr@gmail.com 